INDICATIONS AND USAGE: For the relief of moderate to moderately severe pain. CONTRAINDICATIONS: Hypersensitivity to acetaminophen or hydrocodone WARNINGS: Respiratory Depression: At high doses or in sensitive patients, hydrocodone may produce dose-related respiratory depression by acting directly on the brain stem respiratory center. Hydrocodone also affects the center that controls respiratory mythm, and may produce irregular and periodic breathing. Head Injury and Increased Intracranial Pressure: The respiratory depressant effects of narcotics and their capacity to elevate cerebrospinal fluid pressure may be markedly exaggerated in the presence of head injury, other intracranial lesions or a preexisting increase in intracranial pressure. Furthermore, narcotics produce adverse reactions which may obscure the clinical course of patients with head injuries. Acute Abdominal Conditions: The administration of narcotics may obscure the diagnosis or clinical course of patients with acute abdominal condtions. PRECAUTIONS. Special Risk Patients. As with any narcotic analgesic agent, Lorcet $10 / 650$ should be used with caution in elderly or debilitated patients and those with severe impairment of hepatic or renal function, hypothy roidism, Addison's disease, prostatic hypertrophy or urethral stricture. The usual precautions should be observed and the possibility of respiratory depression should be kept in mind. Cough Reflex. Hydrocodone suppresses the cough reflex; as with all narcotics, caution should be exercised when Lorcet $10 / 650$ is used postoperatively and in patients with pulmonary disease. Drug Interactions: Patients receiving other narcotic analgesics, antipsychotics, antianxiety agents, or other CNS depressants (including alcohol) concomitantly with Lorceto $10 / 650$ may exhibit an additive CNS depression. When combined therapy is contem plated, the dose of one or both agents should be reduced. The use of MAO inhibitors or tricyclic antidepressants with hydrocodone preparations may increase the effect of either the antidepressant or hydrocodone. The concurrent use of anticholinergics with hydrocodone may produce paralytic ileus. Usage in Pregnancy: Teratogenic Effects: Pregnancy Category C. Hydrocodone has been shown to be teratogenic in hamsters when given in doses 700 times the human dose. There are no adequate and well-controlled studies in pregnant women. Lorcet $10 / 650$ should be used during pregnancy only if the potential benefit justifies the potential risk to the fetus. Nonteratogenic Effects: Babies born to mothers who have been taking opioids regularly prior to delivery will be physically dependent. The withdrawal signs include irritability and excessive crying, tremors, hyperactive reflexes, increased respiratory rate, increased stools, sneezing, yawning, vomiting, and fever. The intensity of the syndrome does not always correlate with the duration of maternal opioid use or dose. There is no consensus on the best method of managing withdrawal. Chlorpromazine 0.7 to $1 \mathrm{mg} / \mathrm{kg} \mathrm{q} 6 \mathrm{~h}$, and pare goric 2 to $4 \mathrm{drops} / \mathrm{kg}$ q $4 \mathrm{~h}$, have been used to treat withdrawal symptoms in infants. The duration of therapy is 4 to 28 days, with the dosage decreased as tolerated. Labor and Delivery: As with all narcotics, administration of Lorcet $10 / 650$ to the mother shortly before delivery may result in some degree of respiratory depression in the newborn, especially if higher doses are used. Nursing Mothers. It is not known whether this drug is excreted in human milk. Because many drugs are excreted in human milk and because of the potential for serious adverse reactions in nursing infants from Lorcet ${ }^{\circ} 10 / 650$, a decision should be made whether to discontinue nursing or to discontinue the drug, taking into ac count the importance of the drug to the mother. Pediatric Use: Satety and effectiveness in chidren have not been established. ADVERSE REACTIONS: The mOS frequently observed adverse reactions include lightheadedness, dizziness, sedation, nausea and vomiting. These effects seem to be more prominent in ambula. tory than in nonambulatory patients and some of these adverse reactions may be alleviated if the patient lies down. Other adverse reactions include: Central Nervous System: Drowsiness, mental clouding, lethargy, impairment of mental and physical performance, anxiety, tear, dysphoria, psychic dependence, mood changes. Gastrointestinal System: The antiemetic phenothiazines are useful in suppressing the nausea and vomiting which may occur (see above); however, some phenothiazine derivatives seem to be antianalgesic and to increase the amount of narcotic required to produce pain relief, while other phenothiazines reduce the amount of narcotic required to produce a given level of analgesia. Prolonged administration of Lorcet $10 / 050$ may produce constipation. Genito urinary System: Ureteral spasm, spasm of vesical sphincters and urinary retention have been reported. Respiratory Depression: Hydrocodone bitartrate may produce dose-related respiratory depression by acting directly on the brain stem respiratory center. Hydrocodone also alfects the center that controls respiratory rhythm, and may produce irregular and periodic breathing. If significant respiratory depression occurs, it may be antagonized by the use of naloxone hydrochlo ride. Apply other supportive measures when indicated. ORUG ABUSE AND DEPENDENCE: Lorcet 10/650 is subject to the Federal Controlled Substances AC (Schedule III). Psychic dependence, physical dependence, and tolerance may develop upon repeated administration of narcotics; therefore, Lorcet $10 / 650$ should be prescribed and administered with caution. However, psychic dependence is unilikely to develop when Lorcet $10 / 650$ is used for a short time for the treatment of pain. OVERDOSAGE. Acetaminophen: Signs and Symptoms: In acute acetaminophen overdosage, dose-dependent, potentially fatal hepatic necrosis is the most serious adverse effect. Renal tubular necrosis, hypoglycemic coma, and thrombocytopenia may also occur. Early symptoms following a potentially hepatotoxic overdose may include: nausea, vomiting, diaphoresis and general malaise. Clinical and laboratory evidence of hepatic toxicity may not be apparent until 48 to 72 hours post-ingestion. Hydrocodone: Signs and Symptoms. Serious overdose with hydrocodone is characterized by respiratory depression (a decrease in respiratory rate and/or tidal volume, Cheyne-Stokes respiration, cyanosis), extreme somnolence progressing to stupor or coma, skeletal muscle flac cidity, cold and clammy skin, and sometimes bradycardia and hypotension. In severe overdosage, apnea, circulatory collapse, cardiac arrest and death may occur. DOSAGE AND ADMINISTRATION: Dosage should be adjusted according to the severity of the pain and the response of the patient. However, it should be kept in mind that tolerance to hydrocodone can develop with continued use and that the incidence of untoward effects is dose related. The usual adult dosage is one tablet every four to six hours as needed for pain. The total 24 hour dose should not exceed 6 tablets. CAUTION: Federal law prohibits dispensing without prescription. A Schedule Clit Controlled Substance. Manufactured by: MIKART INC. ATLANTA, GA 30318 Manufactured for UAD Laboratories Division of
Forest Pharmaceuticals, Inc. St. Louis, MO 63045 Rev. 6/94 Code 558A00

9. FOREST PHARMACEUTICALS, INC.

UAD LABORATORIES

\title{
Long-term narcotic use may complicate treatment for depression
}

\section{To the Editor:}

I have evaluated and treated numerous patients meeting the diagnostic criteria for major depressive disorder (MDD) with comorbid pain syndrome, the result of having undergone two or more failed spinal surgeries or the result of reflex sympathetic dystrophy. Before psychiatric referral, these patients failed to respond to extensive treatment with physical therapy and/or various medical agents, including nerve blockers, epidural agents, and nonsteroidal anti-inflammatory drugs (NSAIDs), muscle relaxants, and narcotics.

Those patients not taking narcotics, but taking NSAIDs and a muscle relaxant, respond more quickly and more fully to antidepressant treatment than do those patients who are receiving narcotic therapy for an extended period. The patients taking narcotics often have inadequate responses to treatment for depression, despite being on therapy with various antidepressants and augmentary agents. The patients' histories and presenting physical symptoms do not consistently establish that patients on long-term narcotic therapy are more physically ill than patients not taking narcotics.

Narcotics may alter mood and cause mental confusion,,$^{1,2}$ and with extended use, diminish the patient's pain threshold. Although these agents may be efficacious for shortterm use, over a long period, significant reason exists to question their danger. It appears difficult to wean patients off narcotics once they have taken these drugs for years.

As osteopathic physicians, we are empathic to the misery of our patients with chronic pain. There are patients who may require longterm narcotic therapy. This commentary is not to discredit their need, but to emphasize the potentially serious negative psychiatric consequences of such a regimen. Physicians should consider aggressive psychiatric management of a chronic pain state and carefully weigh the risk-benefit ratio of narcotics with the potential of iatrogenically induced problems, such as depression, mental confusion, and worsening pain states, especially with long-term narcotics use. In particular, the very vulnerable patients with MDD and comorbid pain disorder seem at risk of deterioration without careful assessment of their overall management, including how that management may affect their mood disorder.

Edward H. Tobe, DO Marlton, NJ

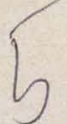

\section{References}

1. France RD, Krishnan KRR:Chronic Pain. Washington, DC, American Psychiatric Press, Inc, 1988, pp 424, 428.

2. Gilman AG, Goodman LS, Rall TW, Murad F (eds): Goodman and Gilman's The Pharmacological Basis of Therapeutics, ed 7. New York, NY, Macmillan Publishing $\mathrm{Co}, 1985$, p 498. 